\title{
Modified Waterfilling Algorithms for MIMO Spatial Multiplexing with Asymmetric CSI
}

\author{
Amitav Mukherjee, Student Member, IEEE, and A. Lee Swindlehurst, Fellow, IEEE
}

\begin{abstract}
This paper studies the transmit power-multiplexing tradeoff in MIMO systems when two users with different priorities are to be accommodated simultaneously. In the general problem formulation, a minimum information rate must be guaranteed for a prioritized user whose channel state information (CSI) is known, while the data rate for the other user with completely unknown CSI is "best-effort." The drawbacks of the classic spatial water-filling algorithm are discussed, and two modified water-filling algorithms are proposed that either balance the required transmit power with the number of spatial dimensions available for each user, or minimize the number of transmit spatial dimensions allocated for the prioritized user. We then specialize to applications in the MIMO wiretap channel, the MIMO multiuser downlink channel, and spectrum underlay in MIMO cognitive radio networks, where existing methods are not applicable under the asymmetric CSI scenario. Numerical results verify the performance advantages of the proposed transmission schemes for each of these case studies.
\end{abstract}

Index Terms-MIMO, resource allocation, waterfilling.

\section{INTRODUCTION}

$\mathbf{T}$ HE classic waterfilling algorithm and its generalized variants are well-known solutions to numerous optimization problems encountered in single- and multi-user communication theory, most often related to the maximization of mutual information [1]. However, in this work we consider problems of a dual nature in a multiple-input multiple-output (MIMO) setting, such as providing a minimum guaranteed information rate to a prioritized user for a given transmit power constraint and antenna array size, while using any remaining resources to serve lower-priority users. We demonstrate in this work that the classic waterfilling (CWF) algorithm does not always make the most efficient use of the available resources, and propose two suitable alternatives to this end, namely, "balanced" and "frugal" waterfilling. It is interesting to note that while the diversity-multiplexing tradeoff for MIMO systems has been studied extensively [2], the power-multiplexing tradeoff has received comparatively lesser attention. In related work, the multi-antenna broadcast channel with single-antenna besteffort and delay-constrained receivers was studied in [3], and a combination of user and antenna selection was proposed to obtain the number of data streams that maximizes throughput assuming uniform power allocation over all streams. However, our proposed algorithms attempt to optimize both power and data stream allocation, and are applicable to a wide range of multi-antenna networks as shown in the sequel.

Manuscript received December 2, 2011. The associate editor coordinating the review of this letter and approving it for publication was K. Huang.

This work was supported by the U.S. ARO under MURI grant W911NF-071-0318, and by the National Science Foundation under grant CCF-1117983.

The authors are with the Dept. of EECS, University of California, Irvine, CA 92697-2625, USA (e-mail: \{amukherj, swindle\}@uci.edu).

Digital Object Identifier 10.1109/WCL.2012.012712.110227

\section{Mathematical Model}

\section{A. Transmission Scheme}

The general transmission model is as follows. We consider a multi-antenna transmitter equipped with $N_{a}$ antennas, which transmits a signal vector $\mathbf{x}_{a} \in \mathbb{C}^{N_{a} \times 1}$, to one or more multi-antenna receivers. The transmit power is assumed to be bounded,

$$
\mathcal{E}\left\{\mathbf{x}_{a} \mathbf{x}_{a}^{H}\right\}=\mathbf{Q}_{a} \quad \operatorname{Tr}\left(\mathbf{Q}_{a}\right) \leq P_{a},
$$

where $\mathcal{E}\{\cdot\}$ denotes expectation, $(\cdot)^{H}$ the Hermitian transpose, and $\operatorname{Tr}(\cdot)$ denotes the trace operator.

The transmit signal is split into two components, a highpriority message with a pre-specified QoS (rate) constraint, denoted by the $N \times 1$ vector $\mathbf{z}$, and a $\left(N_{a}-N\right) \times 1$ besteffort data vector $\mathbf{z}^{\prime}$. The term "best-effort" here refers to the presence of a secondary or lower-priority goal to be addressed in addition to achieving a desired rate for the prioritized receiver. Let $0<\rho \leq 1$ denote the fraction of the total available power devoted to $\mathbf{z}$, and let $\mathbf{T}, \mathbf{T}^{\prime}$ represent the $N_{a} \times N$ and $N_{a} \times\left(N_{a}-N\right)$ precoders corresponding to $\mathbf{z}$ and $\mathbf{z}^{\prime}$ :

$$
\mathbf{x}_{a}=\mathbf{T} \mathbf{z}+\mathbf{T}^{\prime} \mathbf{z}^{\prime}
$$

The elements of $\mathbf{z}$ are assumed to be uncorrelated, and the vectors $\mathbf{z}$ and $\mathbf{z}^{\prime}$ are uncorrelated with each other as well. Thus, $\mathbf{Q}_{a}$ may be expressed as

$$
\mathbf{Q}_{a}=\mathbf{T} \mathbf{Q}_{z} \mathbf{T}^{H}+\mathbf{T}^{\prime} \mathbf{Q}_{z}^{\prime} \mathbf{T}^{\prime H}
$$

where $\mathbf{Q}_{z}, \mathbf{Q}_{z}^{\prime}$ are covariance matrices associated with $\mathbf{z}$ and $\mathbf{z}^{\prime}$, respectively, such that $\operatorname{Tr}\left(\mathbf{T} \mathbf{Q}_{z} \mathbf{T}^{H}\right) \leq \rho P_{a}$, and $\operatorname{Tr}\left(\mathbf{T}^{\prime} \mathbf{Q}_{z}^{\prime} \mathbf{T}^{\prime H}\right) \leq(1-\rho) P_{a}$.

We assume that the transmitter has complete knowledge of the channel to the prioritized receiver, but no information whatsoever (either instantaneous or statistical) of the channel to the best-effort receiver. This is denoted as the asymmetric CSI scenario at the transmitter. Therefore, we are restricted to transmit precoding operations such as waterfilling on the prioritized channel alone. If we let $\mathbf{H}=\mathbf{U} \boldsymbol{\Sigma} \mathbf{V}^{H}$ denote the singular value decomposition of the MIMO channel to the prioritized receiver, then the precoder $\mathbf{T}$ for the primary signal will be chosen as the $N$ most dominant right singular vectors of $\mathbf{H}$, which we denote by $\mathbf{T}=\mathbf{V}(:, 1: N)$. Furthermore, we will constrain the secondary signal so that it does not cause any interference to the prioritized receiver. To guarantee this, we require

$$
\mathbf{H T z} \perp \mathbf{H T}^{\prime} \mathbf{z}^{\prime}
$$

for all possible $\mathbf{z}$. This goal can be achieved by choosing $\mathbf{T}^{\prime}$ to be the remaining $N_{a}-N$ right singular vectors: $\mathbf{T}^{\prime}=\mathbf{V}($ : $\left., N+1: N_{a}\right)$. 


\section{B. Modified Waterfilling}

Assuming no interference other than Gaussian noise of variance $\sigma^{2}$ at the primary receiver, a target data rate of $R_{b}$ over the associated MIMO channel $\mathbf{H}$ is achieved by choosing $\rho, N$ and $\mathbf{T}$ so that

$$
R_{b}=\log \left|\mathbf{I}+\mathbf{H T} \mathbf{Q}_{z} \mathbf{T}^{H} \mathbf{H}^{H} / \sigma^{2}\right| .
$$

Ideally, one would like to achieve (4) using the smallest amount of transmit power possible, so that all remaining power could be devoted to serving best-effort traffic. However, under the classic waterfilling algorithm the lowest transmit power is achieved when $N$ is chosen as large as possible (up to $\left.\operatorname{rank}(\mathbf{H})=\min \left(N_{b}, N_{a}\right)\right)$. Depending on the power distribution strategy, larger values of $N$ may result in certain transmit beamformers being allocated a relatively small fraction of the total transmit power, which results in an inefficient use of those spatial dimensions and starvation of the best-effort receivers.

Therefore, an intuitive strategy to avoid the above problem is to conduct a Frugal Waterfilling (FWF) operation which simply seeks to minimize the number of transmit spatial dimensions $N$ needed to achieve $R_{b}$. However, minimizing the spatial footprint of the prioritized user via FWF inevitably leads to a higher transmission power compared to CWF, and subsequently diminished transmit power for the best-effort data.

Based on these observations, we also propose a modified power-multiplexing allocation strategy referred to as Balanced Waterfilling (BWF), that attempts to balance the competing objectives of power and spatial dimensions by minimizing the product $\rho N$. In BWF, the use of additional channels to achieve the desired rate effectively incurs a penalty proportional to the number of channels used. This is evidently a heuristic approach and numerous other 'penalty' terms can be applied to the waterfilling objective function. In the next section, it is shown that for a broad range of wireless networks, either $B W F$ or FWF can lead to more desirable global performance compared to $C W F$.

The algorithmic description for BWF and FWF is outlined below, where in each case a simple line search together with the classic waterfilling algorithm can be used to solve the problems of interest. In brief, both methods cycle through the possible number of transmit dimensions in ascending order, and at each step compute the transmit power required to meet the rate constraint based on CWF, if feasible. The BWF technique selects the power/dimension combination with the minimum product, whereas the FWF solution chooses the smallest feasible number of transmit dimensions $N$. If $N_{a}=N$ for any of the waterfilling schemes, then all transmit degrees of freedom have been exhausted in achieving the desired QoS, and no best-effort traffic is served.

\section{ApPLiCATIONS}

We now highlight several applications where BWF and FWF offer a performance advantage over classic waterfilling.

\section{A. MIMO Wiretap Channel}

There has been recent significant interest in the three-user wiretap channel, where a transmitter attempts to communicate

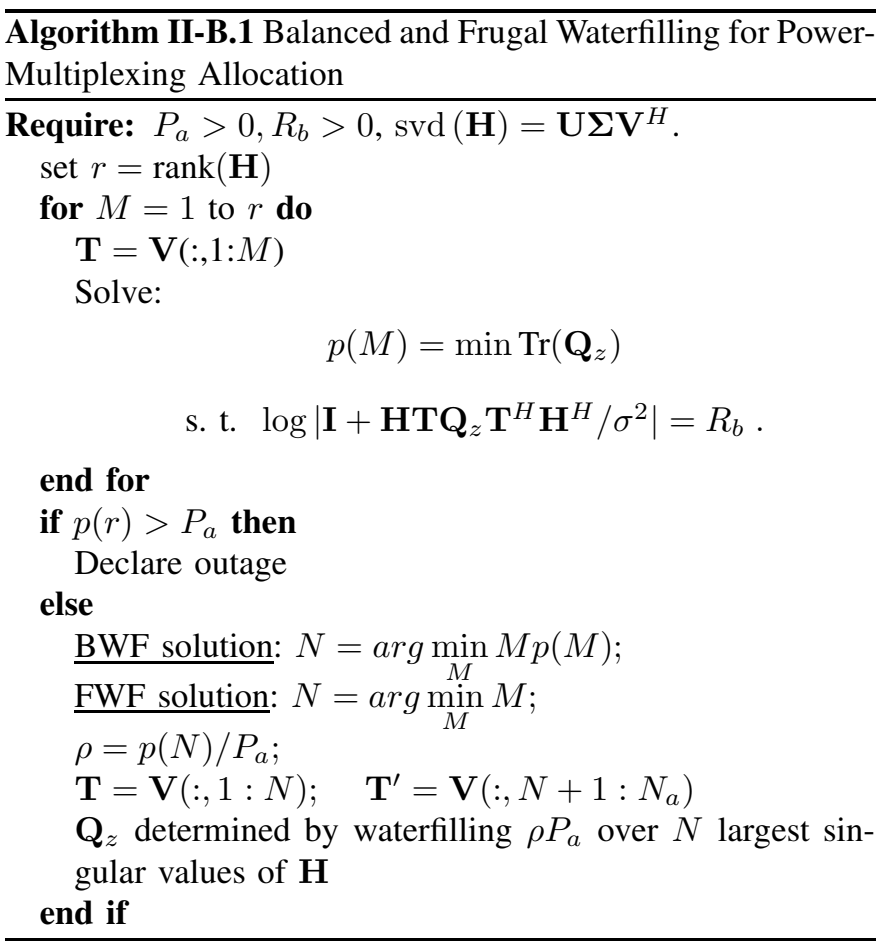

in secret with its receiver in the presence of a passive eavesdropper. In particular, this work led to the development of the notion of secrecy capacity, which quantifies the rate at which a transmitter can reliably send a secret message to the receiver, without the eavesdropper being able to decode it [4].

In particular, one mechanism to increase the security of wireless communications links when the channel state of the eavesdropper is unknown is by transmitting 'artificial interference' along with the confidential data [4]-[6]. The idea is to increase the interference seen by the eavesdropper in such a way that her channel is degraded while the channel of the receiver is not.

In a flat-fading environment, the received signals at the intended receiver and eavesdropper are

$$
\begin{aligned}
& \mathbf{y}_{b}=\mathbf{H}_{b a} \mathbf{x}_{a}+\mathbf{n}_{b} \\
& \mathbf{y}_{e}=\mathbf{H}_{e a} \mathbf{x}_{a}+\mathbf{n}_{e},
\end{aligned}
$$

where $\mathbf{H}_{b a}$ and $\mathbf{H}_{e a}$ are $N_{b} \times N_{a}$ and $N_{e} \times N_{a}$ fading channel matrices, and $\mathbf{n}_{b}, \mathbf{n}_{e}$ are uncorrelated Gaussian noise vectors. In [5], it was shown that for Gaussian input signals and background noise, an achievable secrecy rate for the MIMO wiretap channel is given by

$$
\begin{aligned}
R_{s e c}= & \log \left|\mathbf{I}+\mathbf{K}_{b}^{-1} \mathbf{H}_{b a} \mathbf{T} \mathbf{Q}_{z} \mathbf{T}^{H} \mathbf{H}_{b a}^{H}\right| \\
& -\log \left|\mathbf{I}+\mathbf{K}_{e}^{-1} \mathbf{H}_{e a} \mathbf{T} \mathbf{Q}_{z} \mathbf{T}^{H} \mathbf{H}_{e a}^{H}\right|, \\
\mathbf{K}_{i}= & \mathbf{H}_{i a} \mathbf{T}^{\prime} \mathbf{Q}_{z}^{\prime} \mathbf{T}^{\prime H} \mathbf{H}_{i a}^{H}+\sigma_{i}^{2} \mathbf{I}, \quad i=b, e .
\end{aligned}
$$

When the transmitter has full knowledge of both $\mathbf{H}_{b a}$ and $\mathbf{H}_{e a}$, a generalized singular value decomposition (GSVD) precoding technique was shown to be near-optimal in [5].

However, in our formulation, we consider the important case where no information (instantaneous or statistical) regarding the eavesdropper's channel is available to the transmitter. Without any information about $\mathbf{H}_{e a}$, the secrecy capacity cannot be optimized directly and conventional secure stochastic 
encoding and GSVD precoding is not applicable, but (7) can still be used to quantify the secrecy performance of a given transmission scheme. Therefore, we assign a certain desired information rate $R_{b}$ on the main channel, and devote any remaining resources to the generation of best-effort artificial interference. This approach may not in general yield the best possible performance, since it may be possible to improve the secrecy by allowing some small amount of interference to leak into the legitimate receiver's signal. However, optimization of (7) is difficult and would require knowledge of $\mathbf{H}_{e a}$.

Any of the waterfilling algorithms CWF, BWF or FWF could be used to determine $\rho, \mathbf{Q}_{z}, N$, $\mathbf{T}$, and $\mathbf{T}^{\prime}$ for the MIMO wiretap problem described above. If excess power remains after $R_{b}$ is achieved, then the transmitter simply distributes this as artificial interference with power $(1-\rho) P_{a}$, over these $N_{a}-N$ transmit beamformers:

$$
\mathbf{Q}_{z}^{\prime}=\frac{(1-\rho) P_{a}}{N_{a}-N} \mathbf{I}
$$

\section{B. MIMO Broadcast Channel}

Next, consider a MIMO broadcast channel with a $N_{s^{-}}$ antenna source, $N_{b}$-antenna prioritized receiver with QoS demand $R_{b}$, and a secondary $N_{r}$-antenna receiver. This general scenario captures a multi-antenna cellular downlink [7], or the second hop of a MIMO relay network with multi-priority users, for example. The signals at the receivers are:

$$
\begin{aligned}
\mathbf{y}_{b} & =\mathbf{H}_{s, b} \mathbf{x}_{a}+\mathbf{n}_{b} \\
\mathbf{y}_{r} & =\mathbf{H}_{s, r} \mathbf{x}_{a}+\mathbf{n}_{r}
\end{aligned}
$$

where $\mathbf{x}_{a}$ is defined in (1) as before, $\mathbf{H}_{s, b} \in \mathbb{C}^{N_{b} \times N_{s}}, \mathbf{H}_{s, r} \in$ $\mathbb{C}^{N_{r} \times N_{s}}$, are the associated channel matrices, and $\mathbf{n}_{b}, \mathbf{n}_{r}$ are zero-mean complex Gaussian noise vectors.

The source must ensure that the best-effort data vector does not cause any interference to the high-priority signal. Traditional methods to achieve this aim, such as optimal downlink dirty paper coding (DPC) or block-diagonalization with waterfilling over the effective channels, require the availability of full CSI of both receivers at the transmitter, which is precluded in our system model. Therefore, for our purposes we reuse the construction of $\mathbf{T}, \mathbf{T}^{\prime}$ from orthogonal spaces with uniform power allocation for $\mathbf{z}^{\prime}$ as in Sec. II, thereby allowing the prioritized destination to eliminate interference by projecting into the appropriate subspace.

The prioritized user's signal appears as interference to the best-effort receiver. The covariance of the interference plus noise is given by

$$
\mathbf{K}=\mathbf{H}_{s, r} \mathbf{T} \mathbf{Q}_{z} \mathbf{T}^{H} \mathbf{H}_{s, r}^{H}+\sigma_{r}^{2} \mathbf{I},
$$

and thus the rate achieved for the best-effort user is

$$
R_{h}=\log \left|\mathbf{I}+\mathbf{H}_{s, r} \mathbf{T}^{\prime} \mathbf{Q}_{z}^{\prime} \mathbf{T}^{\prime H} \mathbf{H}_{s, r}^{H} \mathbf{K}^{-1}\right|
$$

Assuming $R_{b}$ is feasible for the prioritized user, the system sum rate is thus

$$
R_{\text {sum }}=\left(R_{b}+R_{h}\right) .
$$

\section{MIMO Cognitive Spectrum Underlay}

Cognitive radios have become renowned as an efficient method of coexistence with legacy networks by means of dynamic spectrum access (DSA). One popular variant of DSA is known as spectrum underlay, where CRs transmit simultaneously with legacy or primary users (PUs), but adapt their transmission parameters so as to confine the interference perceived at the PUs to a pre-specified threshold. This is usually achieved by exploiting knowledge of PU channel state information at the CRs, for example, via beamforming. However, consider the case when the PU CSI is completely unknown at the CR, thereby ruling out the majority of the existing spectrum underlay schemes in the literature [8], [9]. Let the CR's data to its own receiver be designated as the prioritized traffic $\mathrm{z}$ with a target data rate $R_{b}$. This example is a special case of the framework described above with no best-effort traffic being transmitted $\left(\mathbf{T}^{\prime} \mathbf{z}^{\prime}=0\right)$, but we will show that FWF and BWF still afford a potential performance advantage vis-à-vis CWF.

The signal at the PU receiver is given by

$$
\mathbf{y}_{P U}=\mathbf{H}_{1} \mathbf{x}_{P U}+\mathbf{H}_{2} \mathbf{T z}+\mathbf{n}_{P U},
$$

where $\mathbf{H}_{1}, \mathbf{H}_{2}$ are the channels from the PU and CR transmitters (assumed to be full-rank), $\mathbf{x}_{P U}$ and $\mathbf{z}$ are the corresponding transmit signals, and $\mathbf{n}_{P U} \sim \mathbb{C N}\left(\sigma^{2} \mathbf{I}\right)$. The interference covariance matrix at the $\mathrm{PU}$ is $\mathbf{K}=\mathbf{H}_{2} \mathbf{T} \mathbf{Q}_{z} \mathbf{T}^{H} \mathbf{H}_{2}^{H}$. Without knowledge of $\mathbf{H}_{2}$, the CR cannot optimize the PU interference temperature $\operatorname{Tr}(\mathbf{K})$ as in existing DSA systems [8], [9]. However, note that the FWF algorithm guarantees the following:

$$
\operatorname{rank}\left(\mathbf{K}_{F W F}\right) \leq \operatorname{rank}\left(\mathbf{K}_{C W F}\right) .
$$

Therefore, even if $\operatorname{Tr}\left(\mathbf{K}_{F W F}\right)>\operatorname{Tr}\left(\mathbf{K}_{C W F}\right)$, the lowerrank CR interference due to FWF will cause lesser degradation on average to the PU communication link. This is in accordance with well-established results on MIMO capacity, where it is known that reducing the number of transmitted independent data streams alleviates the interference at cochannel receivers, e.g., see [10] and references within.

\section{Numerical Results}

We present some examples that compare the performance of classic and modified waterfilling for various array sizes and example applications. In all simulations, the channel matrices and background noise samples were assumed to be composed of independent, zero-mean Gaussian random variables with unit variance. In situations where the desired rate for the prioritized user cannot be achieved with the given $P_{a}$, rather than indicate an outage, we simply assign all power to the prioritized signal and zero to best-effort data, and average the resulting rate with the others. For each application, we also display the genie performance bound with full CSI, which is unattainable with asymmetric CSI.

Figure 1 illustrates the performance of the algorithms in the MIMO wiretap channel when $R_{b}=8 \mathrm{bits} / \mathrm{s} / \mathrm{Hz}, N_{a}=$ $N_{b}=N_{e}=4$, and the transmit power is varied. In the low to moderate SNR regime, both FWF and BWF provide an improvement of up to $45 \%$ in secrecy rate over CWF. The BWF power allocation ultimately offers the highest secrecy 


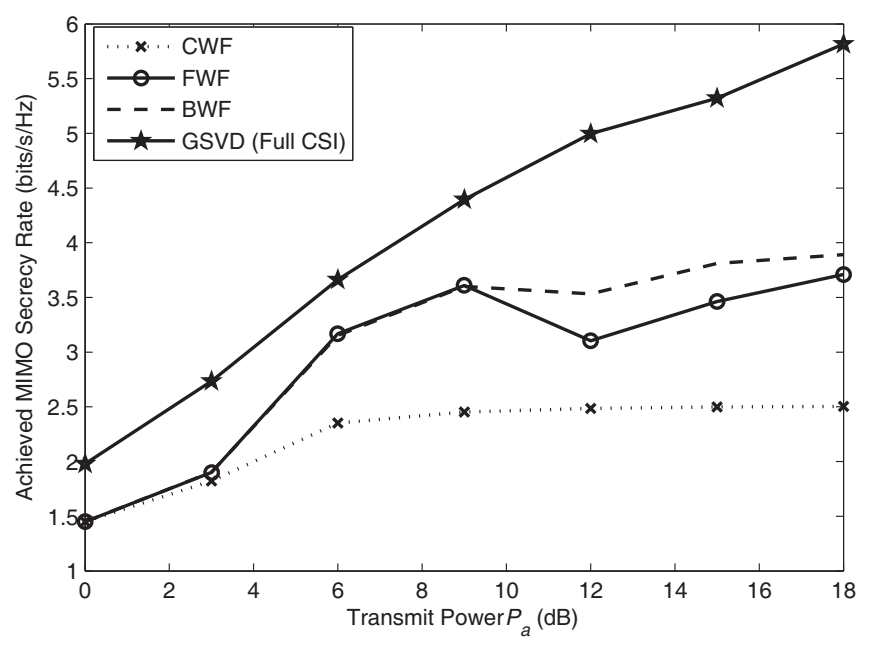

Fig. 1. MIMO secrecy rate versus $P_{a}$ for $N_{a}=N_{b}=N_{e}=4$.

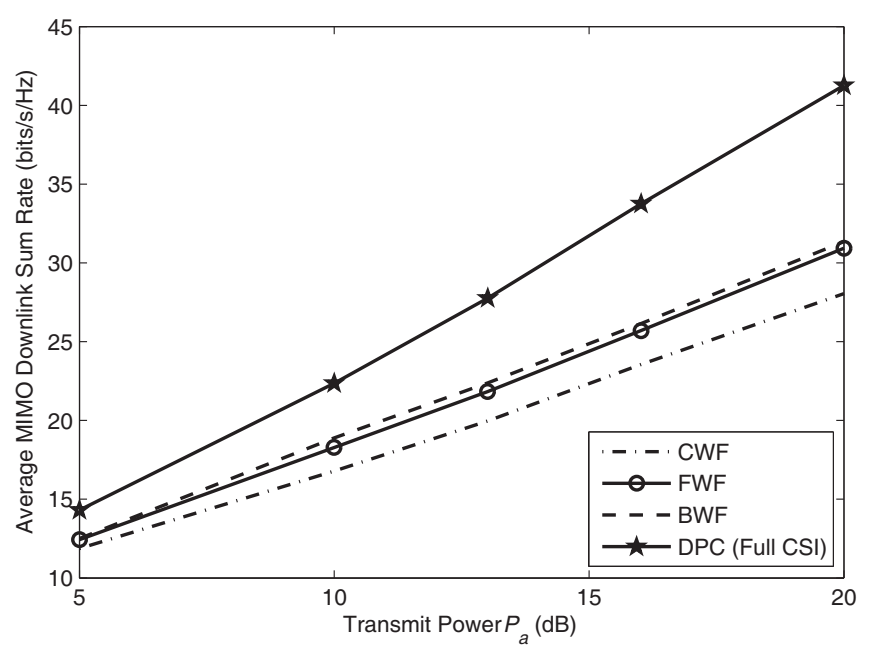

Fig. 2. Two-user MIMO downlink sum rate versus transmit power, for fixed $R_{b}=8 \mathrm{bits} / \mathrm{s} / \mathrm{Hz}$ delivered to prioritized user.

rate at high SNR since it ostensibly reserves additional power for artificial noise.

Figure 2 illustrates the MIMO downlink sum rate as a function of the source transmit power, for a fixed prioritized rate $R_{b}=8 \mathrm{bits} / \mathrm{s} / \mathrm{Hz}$, and six antennas at all terminals. Here, the performance of BWF and FWF is virtually indistinguishable over the entire range of SNR, but is always better than CWF. The critical role of CSI in optimizing multi-user interference at high SNR is emphasized by the widening disparity with SNR between DPC and the asymmetric schemes.

Finally, Figure 3 compares the primary user information rate for a range of desired cognitive user information rates with $P_{a}=75$ and five antennas at all terminals. The genie bound is obtained by minimizing the PU interference temperature while achieving the desired CR rate. The FWF and BWF schemes reduce the spatial footprint of the cognitive radio, and thus in turn reduce the degradation to the PU communication link, with FWF providing up to a $31 \%$ improvement over CWF for less stringent values of $R_{b}$. This gain is achieved even though the CR expends a larger amount of transmit power under FWF compared to CWF, highlighting the importance of low-rank CR interference in spectrum underlay systems.

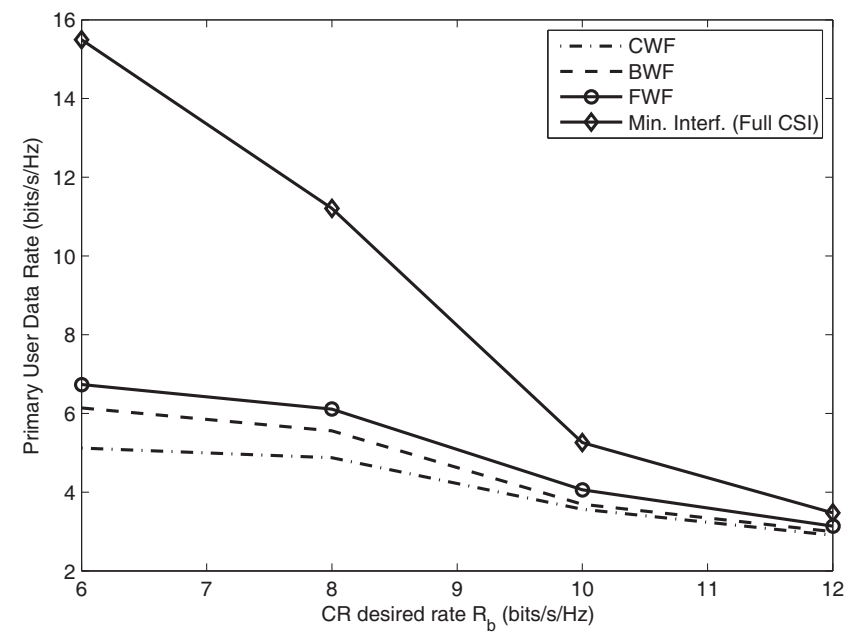

Fig. 3. MIMO primary user data rate versus cognitive radio required rate.

\section{CONCLUSIONS}

We have demonstrated that the conventional MIMO waterfilling algorithm does not typically provide the best performance for a number of applications in MIMO wiretap, multiuser downlink, and cognitive spectrum underlay channels involving data streams with different priorities. Therefore, two modified water-filling algorithms are studied that either balance the required transmit power with the number of spatial dimensions available for each user, or minimize the number of transmit spatial dimensions allocated for the prioritized user. Numerical results for each of these case studies indicate that while FWF and BWF typically outperform CWF, the choice of whether to prefer FWF or BWF is still a open problem.

\section{REFERENCES}

[1] D. P. Palomar and J. R. Fonollosa, "Practical algorithms for a family of waterfilling solutions," IEEE Trans. Signal Process., vol. 53, no. 2, pp. 686-695, Feb. 2005.

[2] L. Zheng and D. Tse, "Diversity and multiplexing: a fundamental tradeoff in multiple-antenna channels," IEEE Trans. Inf. Theory, vol. 49, no. 5, pp. 1073-1096, May 2003.

[3] S.-H. Lee and J. Thompson, "Trade-off of multiplexing streams in MIMO broadcast channels," IEEE Commun. Lett., vol. 14, pp. 115-117, Feb. 2010.

[4] S. Goel and R. Negi, "Guaranteeing secrecy using artificial noise," IEEE Trans. Wireless Commun., vol. 7, no. 6, pp. 2180-2189, June 2008.

[5] A. Khisti and G. Wornell, "Secure transmission with multiple antennas II: the MIMOME wiretap channel," IEEE Trans. Inf. Theory, vol. 56, no. 10, pp. 3471-3475, Oct. 2010.

[6] A. Mukherjee and A. L. Swindlehurst, "Fixed-rate power allocation strategies for enhanced secrecy in MIMO wiretap channels," in Proc. 2009 SPAWC.

[7] A. Vakili, A. Dana, M. Sharif, and B. Hassibi, "Differentiated rate scheduling for MIMO Gaussian broadcast channels," in Proc. 2005 Allerton Conf.

[8] R. Zhang and Y. C. Liang, "Exploiting multi-antennas for opportunistic spectrum sharing in cognitive radio networks," IEEE J. Sel. Topics Signal Process., vol. 2, no. 1, pp. 88-102, Feb. 2008.

[9] K. Hamdi, W. Zhang, and K. B. Letaief, "Opportunistic spectrum sharing in cognitive MIMO wireless networks," IEEE Trans. Wireless Commun., vol. 8, no. 8, pp. 4098-4111, Aug. 2009.

[10] J. Andrews, W. Choi, and R. W. Heath, "Overcoming interference in spatial multiplexing MIMO wireless networks," IEEE Wireless Commun. Mag., vol. 14, no. 6, pp. 95-104, Dec. 2007. 